Publisher: Research Council of Science and Technology, Biratnagar, Nepal p.114

\title{
BIBECHANA
}

A Multidisciplinary Journal of Science, Technology and Mathematics

ISSN 2091-0762 (print), 2382-5340 (online)

Journal homepage: http://nepjol.info/index.php/BIBECHANA

\section{A Study of co-existence between the Hubble flow and the random alignments of spin vectors of SDSS galaxies}

\author{
Shiv Narayan Yadav et al. ${ }^{1}$, Walter Saurer ${ }^{2}$, Binil Aryal ${ }^{{ }^{*}}$ \\ ${ }^{1}$ Central Department of Physics, Tribhuvan University, Kirtipur \\ ${ }^{2}$ Institute of Astrophysics, Innsbruck University, Innsbruck, Austria \\ *E-mail: binil.aryal@uibk.ac.at \\ Accepted for publication: December 19, 2014
}

\begin{abstract}
We present a study of spin vector orientation of 14,118 SDSS (Sloan Digital Sky Survey) galaxies having redshift in the range 0.19 to 0.20 (radial velocity $57,000 \mathrm{~km} / \mathrm{s}$ to $60,000 \mathrm{~km} / \mathrm{s}$ ) with respect to galactic coordinate system using 7th data release (2008, October). These galaxies were observed through SDSS telescope of Apache Point Observatory located at New Mexico, USA. The photometric database is made available through our collaboration with institute of Astro-particle physics, Innsbruck University, Austria. We used the 'Godlowskian' method to convert two dimensional data to three dimensional galaxy rotation axes. Our intension is to find out non-random effects in the spatial orientation of galaxies and to check redshift dependence. The expected isotropy distribution curves are obtained by removing the selection effects and performing a random simulation method. The observed and expected polar and azimuthal angle distributions are compared by using three statistical tests- chi-square, auto-correlation and the Fourier. It is found that the spatial orientation of galaxies tend to be oriented randomly with respect to the galactic coordinate system, supporting hierarchy model of galaxy evolution. In few cases we noticed a preference, probably due to the gravitational shearing or tidal effects in the large scale structure. In general, redshift is found to be independent of orientation. Hence, a very good correlation between the Hubble flow (redshift) and the random alignments of spin vectors of SDSS galaxies is noticed.
\end{abstract}

DOI: http://dx.doi.org/10.3126/bibechana.v12i0.11787

(C) 2014 RCOST: All rights reserved.

Keywords: Supercluster - galaxies; evolution - galaxies; statistics - galaxies; general -- astronomical databases.

\section{Introduction}

Evolution and distribution of galaxies is closely related to the physics of the early universe. The universe was very violent in its early epochs, and galaxies grew quickly, evolving by accretion of smaller mass galaxies. The result of this process is left imprinted on the distribution of galaxies in the nearby universe. Galaxies are not isolated objects in space; rather, galaxies are distributed in great cosmic waves of filaments throughout the universe [1]. We can study galaxy evolution by observing 
the galaxy population at different redshifts since the light from high-redshift galaxies was emitted when the universe was younger. Actually, high-redshift galaxies are the progenitors of present-day galaxies, and any changes in the number density or intrinsic properties of galaxies with redshift give us a direct window on the formation and evolution of the galaxy population.

These days with the help of large telescope we can observe galaxies out to redshift beyond six, making possible for us to probe the galaxy population back to a time when the universe was only $10 \%$ of its current age. The spin vector distribution is a property of galaxies in clusters for which theories make different predictions. The 'pancake model' [2,3], predicts that the spin vectors of galaxies tend to lie within the cluster plane. According to the 'hierarchy model' [4], the directions of the spin vectors should be distributed randomly. The 'primordial vorticity theory' $[5,6]$ predicts that the spin vectors of galaxies are distributed primarily perpendicular to the cluster plane. These three theories are contradictory as their predictions are completely as different from each other.

True spatial orientation of angular momentum of galaxies in the large scale structure is one of the most effective ways of testing the galaxy formation scenarios. $\mathrm{Li}$ (1998) model predicts that the global rotation of the universe provide angular momentum to the large scale structure [7]. According to this model, galaxies form in a rotating universe, and acquire angular momentum through global rotation. Godlowski et al. (2003) showed that Li model predicts the existence of a vanishing angular momentum [8]. Their prediction was observationally tested by several authors [9-18] and found the vanishing angular momenta for less massive structures (galaxy groups, poor galaxy clusters, etc) and non-vanshing angular momenta for larger structures (compact groups, rich galaxy clusters, etc). Therefore, it is essential to probe the angular momenta of galaxy clusters directly based on dynamical analysis.

Study of the evolution of galaxies in the large scale structure is vague and fascinating. So, in order to accomplish our work we have made certain objectives, which are: (a) To test homogeneity and isotropic nature of the universe. (b) To study the preferred alignment of the spin vectors of SDSS galaxies using 'position angle - inclination' method [19]. (c) To test the galaxy evolution models, namely Primordial Vorticity theory, Pancake theory and Hierarchy theory. (d) To study the reliability of galactic co-ordinate system while studying the orientation of distant galaxies. (e) To perform numerical simulation by generating $10^{7}$ virtual galaxies in order to find the expected distribution of angular momentum vectors of galaxies. In the present work we are interested to work on SDSS (7th data release) database of galaxies that have radial velocity in the range $57,000 \mathrm{~km} / \mathrm{s}$ to $60,000 \mathrm{~km} / \mathrm{s}$ $(0.19<z$ (redshift $) \leq 0.20)$. We are interested to check whether redshift dependence exist concerning galaxy orientation or not.

\section{Data Compilation}

The first redshift survey was the CfA Redshift Survey, started in 1977 with the initial data collection completed in 1982. More recently, the 2dF (Two Degree Field) Galaxy Redshift Survey determined the large-scale structure of one section of the Universe, measuring redshifts for over 220,000 galaxies; data collection was completed in 2002, and the final data set was released 30 June 2003. The Sloan Digital Sky Survey (SDSS), is ongoing as of 2015 and aims to measure the redshifts of around 3 million objects. SDSS has recorded redshifts for galaxies as high as 0.8 , and has been involved in the detection of quasars beyond $z=6[21]$. 

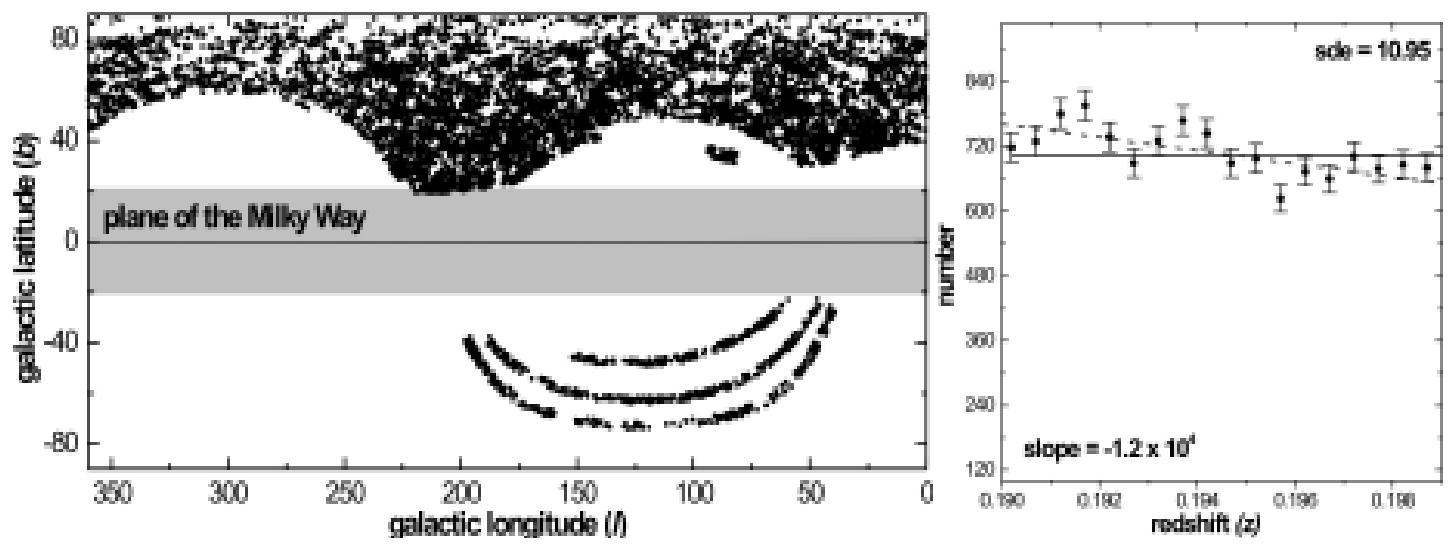

Fig. 1: All sky distribution of galaxies having redshift in the range 0.19 to 0.20 covered by SDSS $7^{\text {th }}$ data release in the galactic co-ordinate system (left). The redshift distribution of galaxies in our database is shown in the right panel. The solid and dashed lines represent expected (average / homogeneous and isotropic) and observed (linear fit / deviation from isotropy) distribution.

We use the database of SDSS galaxies that have redshift in the range 0.19 to 0.20 . These database were downloaded from Sloan Digital Sky Survey (SDSS) Data Release 7[20]. There are 14,118 galaxies in the region of interest. Figure 1 shows the all sky distribution of galaxies of our database. The apparent distribution of the galaxies is because of the nature of the survey. The redshift distribution of galaxies can be seen in Figure 2 (right). The number of galaxies is found to decrease with the redshift. A systematic deviation from the average distribution suggests that the preferred alignments of angular momentum vectors of galaxies are not random. We plotted bin size versus standard error of the database and use basic statistic and obtained the appropriate bin size in order to classify subsamples. We classified total sample into 11 subsamples in order to check deviation from isotropy.

\section{Godlowskian Transformation}

The angular momentum vectors (or spin vectors) of a galaxy is characterized by two angles: the polar angle $(\theta)$ between the angular momentum vectors and a reference plane (here equatorial plane), and the azimuthal angle $(\phi)$ between the projection of a angular momentum vectors on to this reference plane and the $\mathrm{X}$-axis within this plane. The detail derivations of the expressions of the angles $\theta$ and $\phi$ are given in Flin \& Godłowski (1986). When using galactic coordinate system as reference, then $\theta$ and $\phi$ can be obtained from measurable quantities as follows:

$$
\begin{aligned}
& \sin \theta=-\operatorname{cosisin} l \pm \sin i \sin P \cos b \\
& \sin \phi=(\cos \theta)^{-1}[-\cos i \cos b \sin l+\sin i(\mp \sin P \sin b \sin l \mp \cos P \cos l)]
\end{aligned}
$$

where $i$ is the inclination angle, the angle between the normal to the galaxy plane and the observer's line-of-sight. The inclination angle can be computed from the formula 


$$
\cos ^{2} i=\frac{\left(q^{2}-q^{*^{2}}\right)}{\left(1-q^{* 2}\right)}
$$

This expression can be used for oblate spheroids [22], suitable for the disk galaxies [23]. Here, $q$ and $q^{*}$ represent the measured axial ratio (b/a) and the intrinsic flatness of the galaxy, respectively. The intrinsic flatness of a disk galaxy depends on the morphological type and is taken from Heidmann et al. (1971) [24]. Hiedmann et al. (1971) showed that the values of $q^{*}$ range from 0.083 for Sd spirals to 0.33 for ellipticals. For the galaxies with unknown morphology $q^{*}=0.20$ is assumed. In the equations (1) and (2) the galactic longitude, latitude and the position angle is represented by $l, b$ and $P$.

\section{Numerical Simulation}

We briefly explain the procedure by which selection effects can be removed and obtain the expected isotropic distributions for both $\theta$ and $\phi$ as recommended by Aryal \& Saurer (2000) [25]. Theoretically, the isotropic distribution curve for polar angle is cosine and that for azimuthal angle is the average distribution curve, with the restriction that the database is free from se lection effect. Aryal \& Saurer (2000) suggested that any selections imposed on the database may cause severe changes in the shapes of the expected isotropic distribution curves. In their method, a true spatial distribution of the galaxy rotation axis is assumed to be isotropic. Then, due to the projection effects, $i$ can be distributed as $\sin \mathrm{i}, \mathrm{b}$ can be distributed $\sim \cos \mathrm{B}$, the variables 1 and $\mathrm{P}$ can be distributed randomly, and the equation $(1,2)$ can be used to calculate the corresponding values of polar $(\theta)$ and azimuthal $(\phi)$. We run simulations in order to define expected isotropic distribution curves for both the $\theta$ and $\phi$ distributions. The isotropic distribution curves are based on simulations including 107 virtual galaxies. At first we observed the distributions of $1, \mathrm{~b}, \mathrm{P}$ and $\mathrm{i}$ for the galaxies in our samples and distributed by creating 107 virtual galaxies for respective parameters. We use these numbers to make input file and the expected distribution by running simulation in MATLAB 7.0. The program file is as follows:

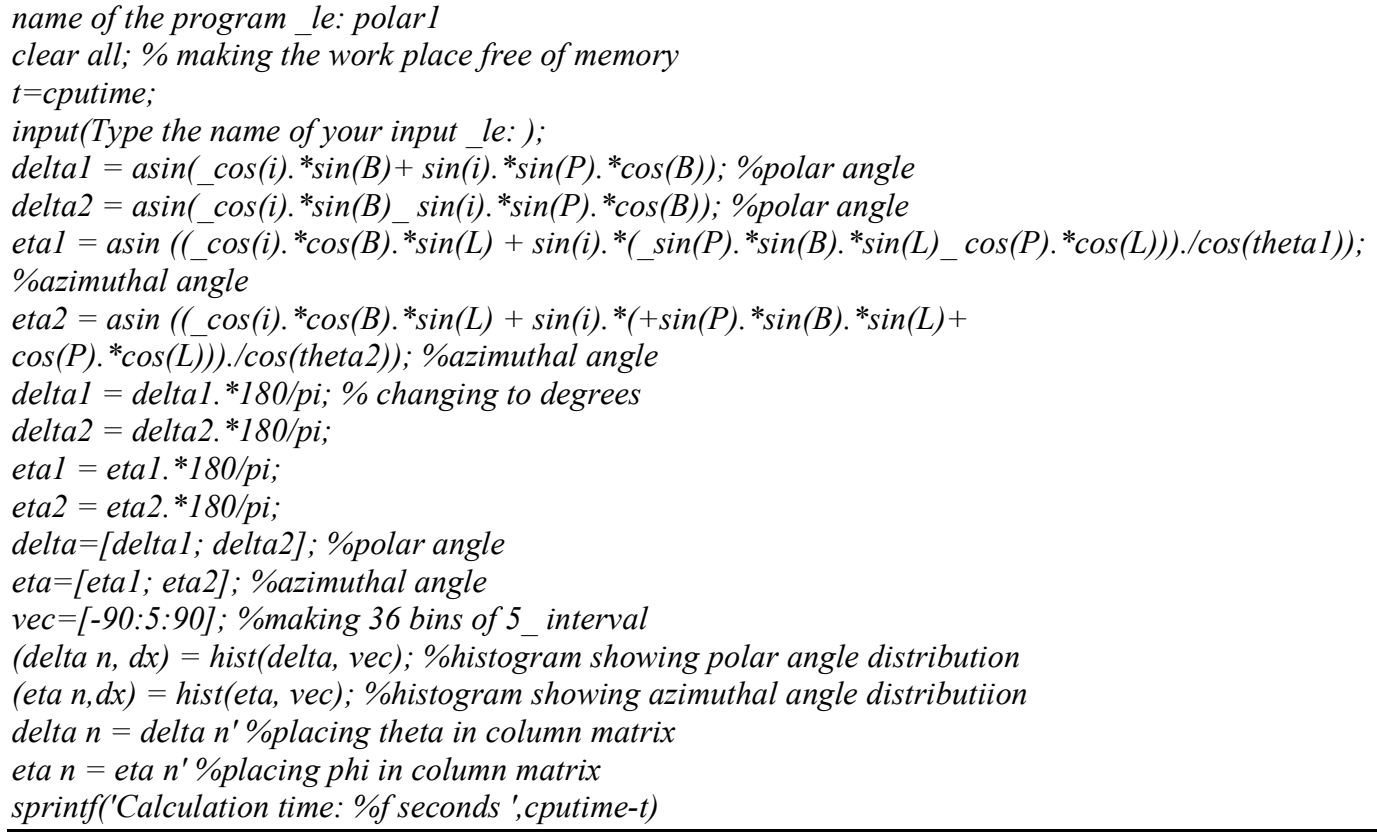




\section{Method of Analysis}

The SDSS dataset of galaxies having redshift in the range 0.19 to 0.20 (real observed dataset) are compared with the isotropic distribution curves (obtained from simulation) in both $\theta$ and $\phi$ distributions. We use three different statistical tests: chi-square-, Fourier-, and auto correlation-test in order to check any deviation from isotropy.

\subsection{Chi-square test}

The chi-square $\chi^{2}$ test is an objective way to examine whether the observational distribution deviates from the expected distribution (in our case, to the isotropic distribution), which is based on the reduced $\chi^{2}$ value given by,

$$
\begin{gathered}
\chi_{v}^{2}=\frac{\chi^{2}}{v} \\
\chi^{2}=\sum_{i=1}^{n} \frac{\left(N_{0 i}-N_{e i}\right)^{2}}{N_{e i}}
\end{gathered}
$$

Here, $n$ represents the number of bins, $N_{o i}$ and $N_{e i}$ represent the observed and expected isotropic distributions and $v$ is the degree of freedom $(v=n-1)$. For an isotropic distribution, the $\chi^{2}$ value is expected to be nearly zero. The quantity $\mathrm{P}\left(>\chi^{2}\right)$ gives the probability that the observed $\chi^{2}$ value is realised by the isotropic distribution. The observed distribution is more consistent with the expected isotropic distribution when $\mathrm{P}\left(>\chi^{2}\right)$ is larger. We set $\mathrm{P}\left(>\chi^{2}\right)=0.050$ as the critical value to discriminate isotropy from anisotropy, it corresponds to the deviation from the isotropy at $2 \sigma$ level.

\subsection{Fourier test}

Fourier test can be applied only if the deviation from isotropy is only slowly varying with $\theta$ or $\phi$. A method of expanding a function by expressing it as an infinite series of periodic functions (sines and cosines) is called Fourier series. The frequencies of the sines and cosines are increased by a constant factor with each successive term. The general mathematical form of the Fourier series is:

$$
f(x)=\frac{a_{0}}{2}+\left(a_{1} \cos x+b_{1} \sin x\right)+\left(a_{2} \cos 2 x+b_{2} \sin 2 x\right)+\cdots
$$

The constants $a_{0}, a_{1}, b_{1}, \ldots \ldots .$. etc., called Fourier coefficients, are obtained by the formulae:

$$
\begin{aligned}
& a_{0}=\frac{1}{\pi} \int_{-\pi}^{\pi} f(x) d x \\
& a_{n}=\frac{1}{\pi} \int_{-\pi}^{\pi} f(x) \cos n x d x \\
& b_{n}=\frac{1}{\pi} \int_{-\pi}^{\pi} f(x) \sin n x d x
\end{aligned}
$$

We compare the observed distributions of the polar and azimuthal angle ( $\theta$ and $\phi)$ of the galaxy rotation axis with the expected isotropic distributions. If the deviation from isotropy is a slowly varying function of the angle $\theta$ (or $\phi$ ), one can use the Fourier test. Let $N$ denote the total number of solutions for galaxies in the sample, $N_{k}$ the number of solutions in the $k^{\text {th }}$ bin, $N_{0}$ the mean number of solutions per bin, and $N_{0 k}$ the expected number of solutions in the $k^{\text {th }}$ bin. Then the Fourier series becomes 


$$
N_{k}=N_{0}\left(1+\Delta_{11} \cos 2 \theta_{k}+\Delta_{21} \sin 2 \theta_{k}+\Delta_{12} \cos 4 \theta_{k}+\Delta_{22} \sin 4 \theta_{k}+\cdots\right)
$$

Here the angle $\theta_{k}$ represents the polar angle in the $k$ th bin. Taking first order Fourier mode,

$$
N_{k}=N_{0}\left(1+\Delta_{11} \cos 2 \theta_{k}+\Delta_{21} \sin 2 \theta_{k}\right)
$$

Here the Fourier coefficients $\Delta_{11}$ and $\Delta_{21}$ are the parameters of the distributions. We obtain the following expressions for the Fourier coefficients $\Delta_{11}$ and $\Delta_{21}$

$$
\begin{gathered}
\Delta_{11}=\frac{\sum_{k=1}^{n}\left(N_{k}-N_{0 k}\right) \cos 2 \theta_{k}}{\sum_{k=1}^{n} N_{0 k} \cos ^{2} 2 \theta_{k}} \\
\Delta_{21}=\frac{\sum_{k=1}^{n}\left(N_{k}-N_{0 k}\right) \sin 2 \theta_{k}}{\sum_{k=1}^{n} N_{0 k} \sin ^{2} 2 \theta_{k}}
\end{gathered}
$$

The standard deviations $\sigma\left(\Delta_{11}\right)$ and $\sigma\left(\Delta_{21}\right)$ can be obtained using the expressions

$$
\begin{aligned}
& \sigma\left(\Delta_{11}\right)=\left(\sum_{k=1}^{n} N_{0 k} \cos ^{2} 2 \theta_{k}\right)^{-1 / 2} \\
& \sigma\left(\Delta_{21}\right)=\left(\sum_{k=1}^{n} N_{0 k} \sin ^{2} 2 \theta_{k}\right)^{-1 / 2}
\end{aligned}
$$

The probability that the amplitude

$$
\Delta_{1}=\left(\Delta_{11}{ }^{2}+\Delta_{21}{ }^{2}\right)^{1 / 2}
$$

is greater than a certain chosen value is given by the formula

$$
P\left(>\Delta_{1}\right)=\exp \left(-\frac{n}{4} N_{0} \Delta_{1}^{2}\right)
$$

with standard deviation

$$
\sigma\left(\Delta_{1}\right)=\left(\frac{2}{n N_{0}}\right)^{1 / 2}
$$

The first order Fourier coefficient $\Delta_{11}$ is extremely useful because it provides the direction of departure from isotropy. In the polar angle $(\theta)$ distribution, if $\Delta_{11}<0$ an excess of galaxies with rotation axis parallel to the reference plane is present, whereas for $\Delta_{11}>0$ the rotation axis tend to be perpendicular to that plane. In the analysis of the azimuthal angle $(\phi)$, if $\Delta_{11}<0$ an excess of galaxies with rotation axis projections perpendicular to the direction of the Virgo cluster center, whereas with $\Delta_{11}>0$ the projections tend to be parallel to that direction. The first order Fourier probability function $P\left(>\Delta_{1}\right)$ explains the depth of the preferred orientation. We set $P\left(>\Delta_{11}\right)=0.150$ as a critical value as suggested by Godlowski (1994) [26].

\subsection{Auto correlation test}

Auto correlation test is a measure of a degree to which there is a linear relationship between two variables. In our case, it takes account the correlation between the number of galaxies in adjoining angular bins. The correlation function is

$$
C=\sum_{1}^{n} \frac{\left(N_{k}-N_{0 k}\right)\left(N_{k+1}-N_{0 k+1}\right)}{\left(N_{0 k} N_{0 k+1}\right)^{1 / 2}}
$$


with the standard deviation $\sigma(C)=(n)^{1 / 2}$. In an isotropic distribution any correlation vanishes, so we expect to have $C \rightarrow 0$

\section{Results and Discussion}

We describe the ways through which polar and azimuthal angle distributions are tested. For this, we use four statistical parameters, namely chi-square probability $\left(\mathrm{P}>\chi^{2}\right)$, autocorrelation coefficient $(\mathrm{C} / \mathrm{C}(\sigma))$, first order Fourier coefficient $\left(\Delta_{11} / \sigma\left(\Delta_{11}\right)\right)$ and first order Fourier probability $\left(\mathrm{P}>\Delta_{1}\right)$. For anisotropy, the limit of chi-square probability $\mathrm{P}\left(>\chi^{2}\right)$ is $<0.050$, auto correlation coefficient $(\mathrm{C} / \mathrm{C}(\sigma))$ is $>1.0$, first order Fourier coefficient $\left(\Delta_{11} / \sigma\left(\Delta_{11}\right)\right)$ is $>1.5$ and Fourier probability $\mathrm{P}\left(>\Delta_{1}\right)$ is $<0.150$ respectively. The statistics for the polar and azimuthal angle distributions is given in Table 1 and Table 2 respectively. In the statistics of $\theta$, a negative value of first order Fourier coefficient suggests that the spin vectors of galaxies tend to be oriented perpendicular with respect to the equatorial coordinate system. Similarly, a positive value of first order Fourier coefficient suggests that the spin vectors of galaxies tend to be oriented parallel with respect to the equatorial coordinate system. Whereas, in the statistics of $\phi$, a positive $\left(\Delta_{11} / \sigma\left(\Delta_{11}\right)\right.$ with significant value suggests that the spin vector projections of galaxies tend to point radially with respect to the center of the equatorial coordinate system. Similarly, a significant negative value of $\left(\Delta_{11} / \sigma\left(\Delta_{11}\right)\right.$ implies that the spin vector projection of galaxies tend to orient tangentially with respect to the equatorial coordinate system.

In addition to the statistical tests, we also study the 'humps' (bins with more solutions than the expected) and 'dips' (bins with less solutions than the expected) in the polar and azimuthal angle distributions. The solid curve, in the histogram of the $\theta$-distribution, represents the expected isotropic distribution whereas dashed curve is the cosine distribution. The solid circles with $\pm 1 \sigma$ error bars represent the observed distribution. The shaded portion represents the range $0^{\circ}<\theta<45^{\circ}$. A hump (or dip) in the smaller $\theta$ suggests that the spin vectors of galaxies tend to orient parallel (or perpendicular) with respect to the equatorial coordinate system.

Table 1: Statistics of the polar (left) and azimuthal angle (right) distributions of SDSS galaxies having redshift in the range 0.19 to 0.20 in the subsamples. The z01 to z11 represents the subsamples as discussed in section 3 . The $\mathrm{P}\left(>\chi^{2}\right)$ represents the chi-square probability (second column). Similarly, $\mathrm{C} / \mathrm{C}(\sigma)$ represents the auto-correlation coefficient (third column). The last two columns give the first order Fourier coefficient $\left(\Delta_{11} / \sigma\left(\Delta_{11}\right)\right.$ and first order Fourier probability $\mathrm{P}\left(>\Delta_{1}\right)$.

\begin{tabular}{|c|c|c|c|c|c|c|c|c|}
\hline \multirow{2}{*}{ Sample } & \multicolumn{4}{|c|}{ Polar Angle $(\theta)$} & \multicolumn{4}{c|}{ Azimuthal Angle $(\phi)$} \\
\cline { 2 - 9 } & $\mathrm{P}\left(>\chi^{2}\right)$ & $\mathrm{C} / \mathrm{C}(\sigma)$ & $\Delta_{11} / \sigma\left(\Delta_{11}\right)$ & $\mathrm{P}\left(>\Delta_{1}\right)$ & $\mathrm{P}\left(>\chi^{2}\right)$ & $\mathrm{C} / \mathrm{C}(\sigma)$ & $\Delta_{11} / \sigma\left(\Delta_{11}\right)$ & $\mathrm{P}\left(>\Delta_{1}\right)$ \\
\hline $\mathrm{z} 01$ & 0.628 & -0.6 & -0.4 & 0.920 & 0.694 & -0.5 & +0.7 & 0.381 \\
\hline $\mathrm{z} 02$ & 0.881 & +0.0 & +0.2 & 0.900 & 0.550 & +0.2 & +0.3 & 0.924 \\
\hline $\mathrm{z} 03$ & 0.781 & +0.1 & +0.0 & 0.834 & 0.661 & -0.1 & +0.2 & 0.367 \\
\hline $\mathrm{z} 04$ & 0.783 & +0.4 & -0.4 & 0.900 & 0.334 & -1.3 & +0.3 & 0.827 \\
\hline $\mathrm{z} 05$ & 0.486 & -0.6 & -0.7 & 0.735 & 0.195 & +1.8 & +2.2 & 0.079 \\
\hline $\mathrm{z} 06$ & 0.310 & -1.7 & +0.1 & 0.921 & 0.224 & +0.5 & +0.6 & 0.768 \\
\hline $\mathrm{z} 07$ & 0.073 & +0.8 & +0.9 & 0.473 & 0.066 & -0.9 & +0.7 & 0.263 \\
\hline $\mathrm{z} 08$ & 0.322 & +1.1 & +1.3 & 0.357 & 0.213 & -1.3 & +0.1 & 0.130 \\
\hline $\mathrm{z} 09$ & 0.266 & -0.6 & +0.5 & 0.764 & 0.606 & +0.6 & +1.7 & 0.096 \\
\hline $\mathrm{z} 10$ & 0.769 & -0.2 & -0.5 & 0.826 & 0.638 & -3.4 & +1.0 & 0.613 \\
\hline $\mathrm{z} 11$ & 0.184 & -0.2 & +1.1 & 0.448 & 0.020 & +2.8 & +1.5 & 0.032 \\
\hline
\end{tabular}


Similarly, a hump (or dip) in the larger $\phi$ indicates that the spin vectors of galaxies tend to be oriented perpendicular with respect to the equatorial coordinate system. In the histogram of the $\phi$-distribution, solid curve represents the expected isotropic distribution whereas dashed curve is the average distribution. The solid circles with $\pm 1 \sigma$ error bars represent the observed distribution. The shaded portion represents the range $-45^{\circ}<\phi<+45^{\circ}$. The humps and dips in the histograms of $\phi$-distribution are not so easy to interpret as compared to $\phi$-distributions. It is because the range of $\phi$ is $-90^{\circ}$ to $+90^{\circ}$. In the histogram of the $\phi$-distribution, $\phi=0^{\circ}$ means spin vector projections tend to point radially towards the center of the equatorial coordinate system. A hump in the middle (central eight bins) of the histogram suggests that the spin vector projections of galaxies tend to point towards the center of the chosen co-ordinate system. Similarly, a hump at first four and last four bins indicates that the spin vectors projections of galaxies tend to be oriented tangentially with respect to the chosen reference coordinate system.

\subsection{Anisotropy in the polar angle distribution}

Table 1 shows the statistics of the polar angle distribution of galaxies in the samples with respect to galactic coordinate system. Figure 2 shows the observed (solid circles with error bars) and expected (solid curves) polar angle distributions.

The statistics for the polar angle distribution in the sample $\mathrm{z} 01$ shows the value of chi-square probability $\left(\mathrm{P}\left(>\chi^{2}\right)\right)$ to be 0.628 i.e. $62.8 \%$ (greater than the significant level 0.050 i.e. $5.0 \%$ ). The auto-correlation coefficient $(\mathrm{C} / \mathrm{C}(\sigma))$ is found to be -0.6 (less than the limit $1 \sigma$ ). The first order Fourier coefficient $\left(\Delta_{11} / \sigma\left(\Delta_{11}\right)\right)$ is found to be -0.4 (less than the limit $1.5 \sigma$ ). The first order Fourier probability $\left(\mathrm{P}\left(>\Delta_{1}\right)\right)$ is found to be 0.921 i.e., $92.1 \%$ (more than $15 \%$ limit). All these statistics suggest isotropy. The polar angle distribution of galaxies in the sample z01 is shown in the Fig. 2a. The number of observed and expected solutions for small angle $\left(<45^{\circ}\right)$ is 1685 and 1679 respectively which suggests that the observed solutions is greater than expected solutions by 6 . There is a significant dip at an angle $12.5^{\circ}$ with $>2 \sigma$ error limit and no hump is notice at all. At bimodal region $\left(\theta \sim 45^{\circ}\right)$, the observed solution is less than expected solution by 2 and the observed and expected distribution agree within $\pm 1 \sigma$. For the large angles $\left(>45^{\circ}\right)$, the expected solution is greater than observed solution by 6 and no humps and dips were seen there. In general, no preferred alignments of spin vectors of galaxies that have redshift in the lower limit is found. This results supports hierarchy model.

Fig. $2 \mathrm{~b}$ shows the $\theta$ distribution for the galaxies of in the sample z02. A dip can be seen at $37.5^{\circ}$ with slightly greater than $\sim 1 \sigma$ error limit. The number of observed solutions for $\theta<45^{\circ}$ is 1728 and expected solutions is 1723 . Thus the number of observed distribution of galaxies is more by 5 than that of the expected. At bimodal region $\left(\theta \sim 45^{\circ}\right)$, the observed solutions is less than the expected solutions by 26. For larger angles $\left(>45^{\circ}\right)$, no humps and dips were observed. The number of observed solutions lag the expected solutions by 5 at $\theta>45^{\circ}$. Statistical parameters for the polar angle distribution of sample z02 indicate isotropy. Thus, a random distribution of angular momentum vectors of galaxies is noticed. The statistics for polar angle distribution for sample z03 (Table 1) suggest strong isotropy. The polar angle distribution of galaxies in the sample z03 is shown in the Fig. 2c. The number of observed and expected solutions for small angle $\left(\theta<45^{\circ}\right)$ is 1583 and 1584 respectively which indicates 1 less solution in observed than the expected distribution. There is dip at 
an angle $7.5^{\circ}$ with equal to $1 \sigma$ error limit and one hump at an angle $47.5^{\circ}$ with slightly $>1 \sigma$ error limit. At bimodal region $\left(\theta \sim 45^{\circ}\right)$, the number of expected solutions is 394 and the number of observed solutions is 418 which shows that the expected solutions is less than the observed solutions by 24 . For the large angles $\left(>45^{\circ}\right)$, the number of observed solution is more by 1 than expected. All statistical tests suggest strong isotropy for z04 (Fig. 2d). A very good agreement between the observed and expected distributions can be seen. At bimodal region $\left(\theta \sim 45^{\circ}\right)$, the observed distribution is less than the expected distribution by 21 . For the $\theta$ less than $45^{\circ}$, the observed distribution is 5 less than the expected whereas for the $\theta$ greater than $45^{\circ}$ the observed distribution is 5 more than the expected.
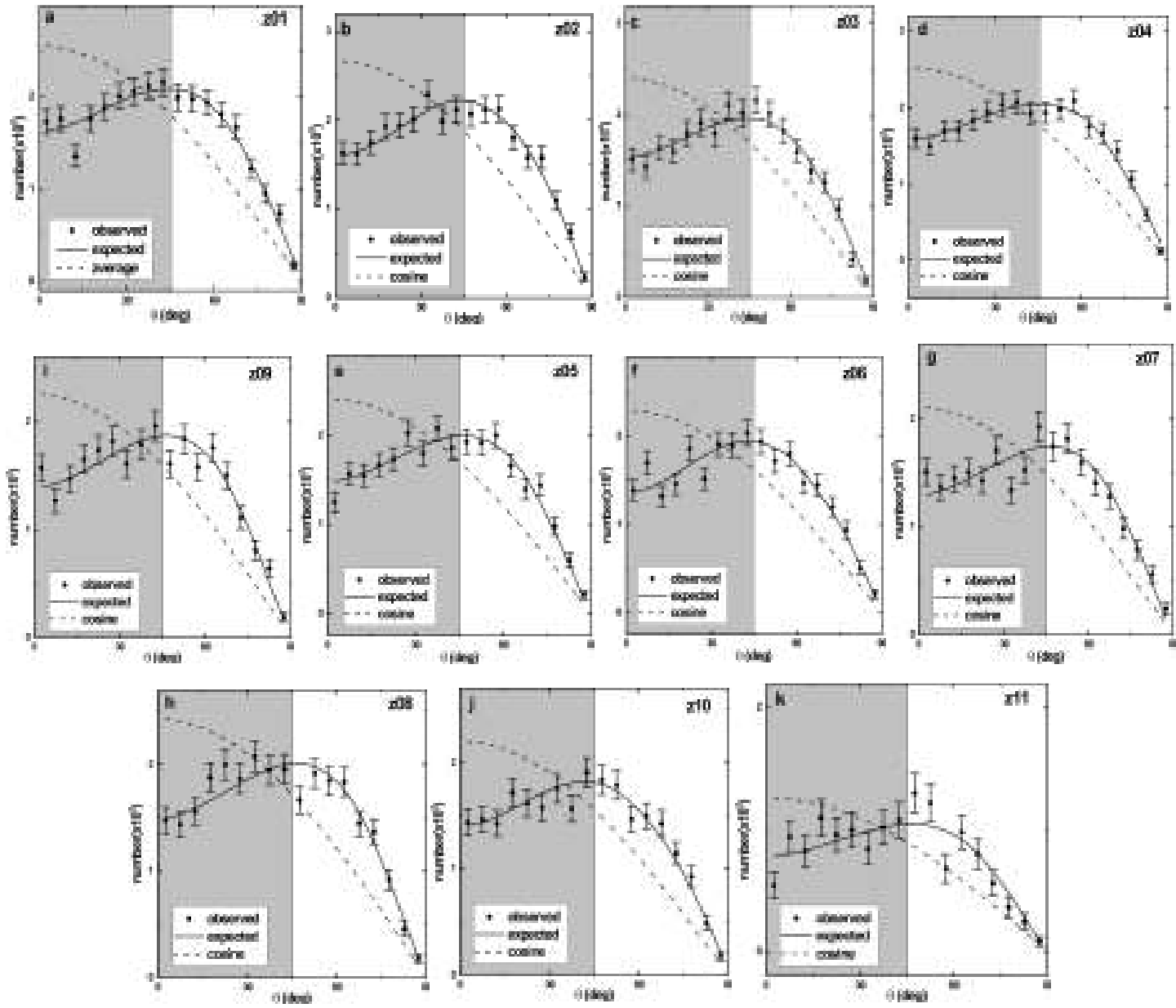

Fig. 2: The polar $(\theta)$ angle distribution of SDSS galaxies in 11 subsamples having redshift in the range 0.19 to 0.20 . The solid line represents the expected isotropic distributions. The cosine distribution (dashed) is shown for the comparison. The statistical error $( \pm 1 \sigma)$ bars are shown for the observed counts.

Similar to the subsample z04, the statistical parameters for z05 suggest strong isotropy. The polar angle distribution of galaxies in the sample z05 is shown in Fig. 2e. There is a dip at an angle $2.5^{\circ}$ with $\sim 2 \sigma$ error and a hump is at an angle $27.5^{\circ}$ with $\sim 1.4 \sigma$ error. At bimodal region $\left(\theta \sim 45^{\circ}\right.$, the 
observed is 21 less than the expected distribution. For the large angles $\left(>45^{\circ}\right)$, the observed distribution is more by 6 than the expected. The statistics for polar angle distribution of sample $\mathrm{z} 06$ is given in Table 1. A strong isotropy is suggested by the all four statistical parameters. The $\theta$ distribution of galaxies is shown in Fig. $2 \mathrm{f}$. For less than $45^{\circ}$, the number of solutions for observed distribution exceeds the expected distribution by 8 . At $7.5^{\circ}$, a hump is seen with $\sim 2 \sigma$ error limit whereas a dip is seen at $27.5^{\circ}$ with $\sim 2 \sigma$ error limit. At bimodal region $\left(\theta \sim 45^{\circ}\right)$, the observed solutions is 10 more than the expected solutions. The number of observes solutions is less than the expected solutions for large angle $\left(>45^{\circ}\right)$ by 7 .

Similar to the samples z01 to z05, a very good agreement between the observed and expected isotropic distribution of galaxies in other samples (z06 to z11) is noticed in the polar angle distribution. This suggests that the spatial orientation of galaxies in the large scale structure prefers hierarchy model as suggested by Peebles (1969) [4].

\subsection{Anisotropy in the azimuthal angle distribution}

Table 1 gives the statistics of the azimuthal angle distribution of galaxies in the samples with respect to galactic coordinate system. Figure 3 shows the observed (solid circles with error bars) and expected (solid curves) azimuthal angle distributions.
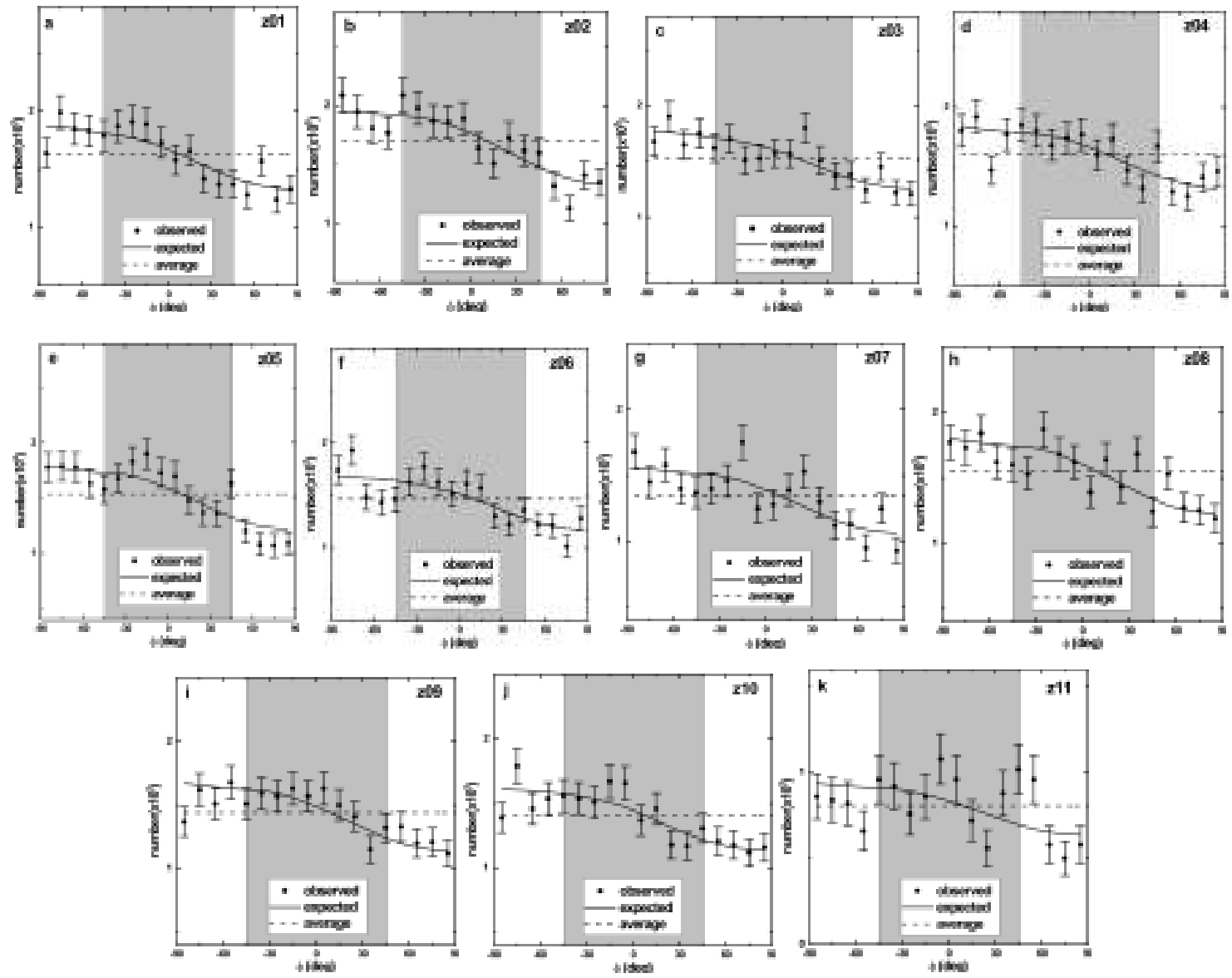

Fig. 3: The azimuthal angle $(\phi)$ distributions of SDSS galaxies in 11 subsamples having redshift in the range 0.19 to 0.20 . The solid line represents the expected isotropic distributions. The cosine and average distribution (dashed) is shown for the comparison. The statistical error $( \pm 1 \sigma)$ bars are shown for the observed counts. 
The statistics for the azimuthal angle distribution (Table 1) in the sample z01 shows the value of chisquare probability $\left(\mathrm{P}\left(>\chi^{2}\right)\right)$ to be 0.694 (greater than the significant level 0.050$)$. The auto-correlation coefficient $\mathrm{C} / \mathrm{C}(\sigma)$ is found to be $-0.5(<1 \sigma)$. The first order Fourier coefficient $\left(\Delta_{11} / \sigma\left(\Delta_{11}\right)\right)$ is found to be 0.7 (less than the limit $1.5 \sigma$ ). The first order Fourier probability $\mathrm{P}\left(>\Delta_{1}\right)$ is found to be $38.1 \%$ (greater than $15 \%$ limit). Here, all the statistics show isotropy. Fig. 3a shows the $\phi$-distribution for the galaxies in the sample z01. A dip is seen at an angle $-85^{\circ}$ and hump is notice at an angle $65^{\circ}$, with nearly $2 \sigma$ error limit. The numbers of observed and expected solutions are 1343 and 1325 respectively for central eight bins. These numbers suggests that the observed distribution exceeds the expected distribution by 18. Overall distributions for the first four bins and last four bins suggest that the observed distribution lags the expected distribution by 10. All the statistics suggest isotropy for $\mathrm{z} 02$. Fig. $3 \mathrm{~b}$ shows the $\phi$-distribution for the galaxies of this sample $\mathrm{z} 02$. At angles $-55^{\circ}$ with $\sim 1.1 \sigma$ error limit, $15^{\circ}$ with $\sim 1.1 \sigma$ error limit and $65^{\circ}$ with $>2 \sigma$ error limit, significant dips are seen and at angle $45^{\circ}$, a significant hump with $\sim 1.1 \sigma$ error limit is seen. For the central eight bins, the observed distribution leads the expected distribution of galaxies by 12. Overall statistics for first four bins and last four bins show the expected distribution leads the observed distribution by 41 . Similar to the subsample z02, all the statistics suggest strong isotropy for z03. From the Fig. 3c, a dip at an angle $25^{\circ}$ with $>1 \sigma$ error is seen. Whereas at an angle $15^{\circ}$, a significant hump is seen $(>2 \sigma)$ and the next hump is seen at an angle $65^{\circ}$ with $>1 \sigma$ error. Statistics for center eight bins indicates that the observed solutions are 6 more than the expected solutions. In the first four bins the expected distributions exceeds the observed distributions by 2 whereas in the last four bins the observed distributions exceeds the expected distributions by 2 .

A strong isotropy is suggested by the statistical parameters for z04 in the azimuthal angle distribution (Fig. 3d). Significance hump is notice at an angle $-65^{\circ}$ with $\sim 3 \sigma$ error limit and at an angle $35^{\circ}$ with $1.1 \sigma$ error limit. Hump is notice at an angle $15^{\circ}$ with equal to $1 \sigma$ error limit and next significance hump is notice at an angle $15^{\circ}$ with $\sim 2 \sigma$ error limit. Statistics reveal 5 less observed solutions than the expected solutions for the center eight bins. For first four and last four bins, the expected distribution leads the observed distribution by 25 . As given in Table 1, statistical parameters for azimuthal angle for sample z05 inferred the value of chi-square probability $\left(\mathrm{P}\left(>\chi^{2}\right)\right)$ is to be $19.5 \%(>5.0 \%)$. The auto-correlation coefficient $\mathrm{C} / \mathrm{C}(\sigma)$ is found to be $1.8(>1 \sigma)$. The first order Fourier coefficient $\left(\Delta_{11} / \sigma\left(\Delta_{11}\right)\right)$ is found to be $2.2(>1.5 \sigma)$. The first order Fourier probability $\mathrm{P}\left(>\Delta_{1}\right)$ is found to be $7.9 \%$ $(<15 \%$ limit). Hence, all the statistics except chi-square probability suggest anisotropy. Fig. 3e shows the $\phi$-distribution for the galaxies of this sample z05. At angles $45^{\circ}$ with $\sim 1.1 \sigma$ error limit, $65^{\circ}$ with $\sim$ $1.2 \sigma$ error limit, $75^{\circ}$ with $\sim 1.2 \sigma$ error limit and $85^{\circ}$ with error limit $\sim 1 \sigma$, significant dips are seen. At an angle $-15^{\circ}$ with error $\sim 2 \sigma$ and at an angle $45^{\circ}$ with error $>2 \sigma$, significant humps are seen. For the central eight bins, the observed distribution leads the expected distribution of galaxies by 44 . In the first four bins the expected distribution is 7 more than the observed and in the last four bins the expected distribution is 52 more than the observed. In this sample, isotropy in the polar angle and anisotropy in the azimuthal angle is seen which shows that our reference galactic coordinate system is inappropriate.

Except in the samples z05 and z09, a random orientation of spin vector projections of galaxies is found in all subsamples. This suggests that the spatial orientation of galaxies in the large scale structure prefers hierarchy model as suggested by Peebles (1969) [4]. 


\section{Conclusion}

We studied the spatial orientation of 14,118 galaxies surveyed by SDSS having redshift in the range 0.19 to 0.20 with respect to the galactic coordinate system. The two dimensional measured parameters are transformed into three dimensional galaxy rotation axes using 'position angle - inclination' method as proposed by Flin \& Godlowski (1986) [19]. In order to remove various selection effects we use a random simulation as proposed by Aryal \& Saurer (2000) [25]. The expected isotropic distribution curves for the total samples are deduced by performing a numerical simulation based on the Godlowskian transformation. Total galaxies are classified into 11 samples on the basis of redshift. To check for anisotropy or isotropy we have carried out three statistical tests: chi-square, auto-correlation and the Fourier. The main goal of this thesis is to examine non-random effects in the galaxy and to find out the appropriateness of choice of coordinate system in order to explain true orientation scenario of distant galaxies. In our work, we have found that, except for samples z05 and z09, our reference galactic coordinate system to explain true orientation scenario was correct. Also our results support hierarchy model of galaxy evolution scenario as suggested by Peebles (1969). We intend to address following issues in our future works.

1. We have studied the galaxy orientation with respect to the galactic coordinate system. We wish to define a local reference system within the galaxies and study the preferred alignments of galaxies in association with it.

2. The results of the statistical analysis have been noticed to have a strong dependence on the sample classification criteria. A straight mathematical computation method instead of statistical method would be essential.

\section{Acknowledgements}

This research has made use of the SDSS database. We acknowledge Prof. Ronald Weinberger for critical discussions. One author (SNY) acknowledges Central Department of Physics, Tribhuvan University, Kirtipur, for providing various forms of support for his doctoral thesis. We thank Mr. Rajesh Kumar Bachchan for his help during data compilation.

\section{References}

[1] T. Padmanabhan, An Invitation to Astrophysics, World Scientific Publishing Co. Pvt. Ltd. (2006). http://dx.doi.org/10.1142/6010

[2] A. G. Doroshkevich, Astrophysical Journ., 14 (1973) L11.

[3] A. G. Doroshkevich, S. F Shandarin, Monthly Notices Royal Astron. Soc., 184 (1978) 643. http://dx.doi.org/10.1093/mnras/184.3.643

[4] P. J. E. Peebles, Astrophysical Journ., 155 (1969) 393. http://dx.doi.org/10.1086/149876

[5] L. M. Ozernoy in M. S. Longair, J Einasto., eds, Proc. IAU Symp. 79, The Large Scale Structure of the Universe. Reidel, Dordrecht (1978).

[6] R. Stein, Astron. \& Astrophys., 35 (1974) 17. 
[7] L. X. Li, General Relativity and Gravitation, 30 (1998) 497. http://dx.doi.org/10.1023/A:1018867011142

[8] W. Godlowski, M. Szydlowski, P. Flin, M. Biernacka, General Relativity and Gravitation, 35 (2003) 907. http://dx.doi.org/10.1023/A:1022959523795

[9] W. Godlowski, M. Szydlowski, P. Flin, General Relativity and Gravitation, 37 (2005) 615. http://dx.doi.org/10.1007/s10714-005-0046-7

[10] B. Aryal, W. Saurer, Astronom. Astrophys., 425 (2004) 871. http://dx.doi.org/10.1051/0004-6361:20041228

[11] B. Aryal, W. Saurer, Astronom. Astrophys., 432 (2005a) 841. http://dx.doi.org/10.1051/0004-6361:20041975

[12] B. Aryal, W. Saurer, Astronom. Astrophys. 432 (2005) 431. http://dx.doi.org/10.1051/0004-6361:20041679

[13] B. Aryal, W. Saurer, Monthly Notices Royal Astron. Soc., 366 (2006) 438. http://dx.doi.org/10.1111/j.1365-2966.2005.09667.x

[14]B. Aryal, S. Paudel, W. Saurer, Astronom. Astrophys., 479 (2008) 397. http://dx.doi.org/10.1051/0004-6361:20077810

[15] B. Aryal, S. N. Yadav, W. Saurer, Bulletin of Astron. Astron. Soc. Ind. (BASI), 40 (2012) 65.

[16] B. Aryal, H. Bhattarai, S. Dhakal, C. Rajbahak, W. Saurer, Monthly Notice of Royal Astron. Soc., 434 (2013) 1939. http://dx.doi.org/10.1093/mnras/stt1124

[17] W. Godlowski, P. Piwowarska, E. Panko, P. Flin, Astrophysical Journ., 723 ( 2) (2010) 985. http://dx.doi.org/10.1088/0004-637X/723/2/985

[18] W. Godlowski , Astrophysical Journ., 747 (1) (2012) 7. http://dx.doi.org/10.1088/0004-637X/747/1/7

[19] P. Flin, W. Godlowski, Monthly Notices Royal Astron. Soc., 222 (1986) 525. http://dx.doi.org/10.1093/mnras/222.3.525

[20] M. Colless et. al., Monthly Notices Royal Astron. Soc., 328 (2001) 1039. http://dx.doi.org/10.1046/j.1365-8711.2001.04902.x

[21] D. G. York, J. Adelman, J.E. Anderson, The Sloan Digital Sky Survey: Technical Summary (2000).

[22] E. Holmberg, Medd. Lund. Astron. Obs., Ser. VI, No. 117 (1946).

[23] W. Godlowski, Monthly Notices Royal Astron. Soc., 265 (1993) 874. http://dx.doi.org/10.1093/mnras/265.4.874 
Yadav et al./BIBECHANA 12 (2015) 114-127: p. 127

[24] J. Heidmann, N. Heidmann, G. de Vaucouleurs, Monthly Notices Royal Astron. Soc. 75 (1971) 85.

[25] B. Aryal, W. Saurer, Astronom. Astrophys. lett., 364 (2000) L97.

[26] W. Godlowski, Monthly Notices Royal Astron. Soc, 271 (1994) 19.

http://dx.doi.org/10.1093/mnras/271.1.19 